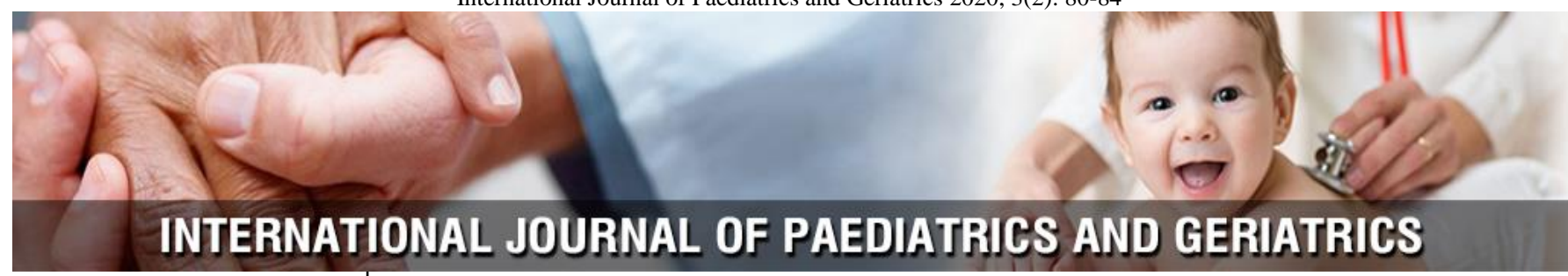

P-ISSN: 2664-3685

E-ISSN: $2664-3693$

IJPG 2020; 3(2): 80-84

Received: 21-05-2020

Accepted: 24-06-2020

Dr. Christy Maria Viagulam J Post Graduate, Department of Pediatrics, Raja Muthiah Medical College,

Chidambaram, Tamil Nadu, India

Dr. Saravanan S

Associate Professor,

Department of Pediatrics,

Raja Muthiah Medical College,

Chidambaram, Tamil Nadu, India

\section{Early initiation of bubble CPAP and its outcome in neonates with respiratory distress}

\author{
Dr. Christy Maria Viagulam J and Dr. Saravanan S
}

DOI: https://doi.org/10.33545/26643685.2020.v3.i2b.99

\begin{abstract}
Background: Respiratory distress in newborn is one of the commonest causes of neonatal mortality and morbidity in many developing countries. The early initiation of Bubble Continuous Positive Airway Pressure, a low-cost and simple technique can treat respiratory distress in an effective manner. We compared and studied the outcome of initiating bubble CPAP, by the $1^{\text {st }}$ hour of birth in neonates with respiratory distress. Bubble CPAP was a success amongst neonates who were of gestational age more than 32 weeks, weighing more than 1200gms and with Downes' score of less than 7.

Methods: The study was conducted in a rural tertiary care hospital amongst 150 neonates admitted in intramural (in born) and extramural (out born) neonatal intensive care units. The study was done over a period 18 months. The primary outcome of the study was to compare the proportion of success or failure on early initiation of bubble CPAP.

Results: In our study $94 \%$ of babies admitted with respiratory distress and started on Bubble CPAP survived while by early initiation in the $1^{\text {st }}$ hour, $80.9 \%$ of babies were treated successfully.

Conclusion: Bubble CPAP has a high percentage of success when initiated early in neonates with mild and moderate respiratory distress
\end{abstract}

Keywords: (CPAP) Continuous Positive airway pressure, (BCPAP) Bubble Positive airway pressure, $\left(\mathrm{FiO}_{2}\right)$ fraction of inspired oxygen

\section{Introduction}

Neonatal mortality is one of the top 5 causes that contributes to global disease burden, in India. Worldwide one of the leading causes of mortality among neonates is respiratory distress. Both preterm and term infants are prone for respiratory distress due to various underlying causes. The term and preterm infantshave differing anatomy and physiology and lung maturity. As the varying causes leading respiratory distress in term and preterm infants are different, they require different modalities of approach and treatment. However all severe forms of respiratory distress requires oxygenation to avoid desaturation, cyanosis, shock or death. Continuous Positive airway pressure (CPAP) was discussed by WHO experts as an alternative to intubation among preterm neonates with respiratory distress. WHO recommends using CPAP, as early intervention provides oxygenation and prevents complications in a newborn with respiratory distress, though the concept requires further study ${ }^{[1,2]}$. Many studies in BCPAP done earlier was on preterm infants as early as $1970 \mathrm{~s}^{[3}$, 4], as CPAP was used extensively for newborn with Respiratory distress syndrome post surfactant. However our study included more of term and late preterm infants, as we receive many neonates with respiratory distress from nearby primary and secondary care institutions. Though oxygen can be provided through continuous positive airway pressure, CPAP can also be provided via various interfaces such as face mask, nasal prongs, mechanical ventilator, CPAP machine and the Bubble CPAP (BCPAP). Bubble CPAP is known for its low cost and its delivery of CPAP by underwater seal ${ }^{[5,9]}$. BCPAP with its underwater seal has been widely researched and implemented across various neonatal intensive care units across the World, especially in low middle income countries ${ }^{[10]}$. It is said that underwater seal causes vibrations in the non compliant chest wall of a neonate. The constant distending pressure keeps the collapsed alveoli patent, thus improving the functional residual capacity and thus increases gas exchange. Though this mechanism was disputed in few studies, research in animal studies ${ }^{[16]}$ showed that BCPAP improved alveolar development in preterm infants, improved lung volume and strengthened peripheral airway patency ${ }^{[6,7]}$, reducing respiratory distress in neonates and was superior to Mechanical ventilation in terms
Corresponding Author:

Dr. Saravanan S

Associate Professor,

Department of Pediatrics,

Raja Muthiah Medical College,

Chidambaram, Tamil Nadu,

India 
of reducing complications ${ }^{[8]}$.

We studied the effects of Bubble CPAP in respiratory distress in early, late preterm and term neonates who had varying etiologies, maturity, birth weight etc. ${ }^{[11,12]}$, The intervention of initiating Bubble CPAP (BCPAP)and its implications, in neonates was classified as success or failure at the end of the study.

\section{Methods}

This comparative study of the final outcome was conducted at neonatal intensive care units of Raja Muthiah Medical College and Hospital. This Tertiary care centre serves as a rural teaching institute with restraints in the form of infrastructure, lab consumables and funding. Though the centre had mechanical ventilators, it was not feasible to provide such equipment to all sick newborn admitted with respiratory distress. Hence as an alternative, the effect of BCPAP was studied in 150 neonates admitted to the intramural (babies born inside the institution) and extramural (babies delivered outside and referred) neonatal intensive care units with respiratory distress. (Table 1)

\section{Exclusion criteria}

1. Neonates with progressive respiratory failure/poor respiratory drive requiring mechanical ventilation

2. Neonates with inability to maintain oxygenation or with severe hypotension or circulatory failure (shock)

3. Neonates with congenital malformations of airway like cleft palate, choanal atresia, Tracheoesophageal fistula, surgical conditions

4. Neonates with congenital heart disease

5. Post surfactant administered neonates

All neonates with respiratory distress were classified as mild, moderate and severe based on Downes scoring. The score had 5 criteria namely respiratory rate, retractions, grunting, air entry and cyanosis. A score of 0 to 2 was assigned for each parameter and the final score, mild $(<5)$, moderate (5-7) and severe (>7) indicated the severity of distress. Chest Xray was used to identify the underlying cause and to rule out complications. Blood investigations such as complete blood hemogram, renal functional tests and electrolytes were taken. Babies who had sepsis were in turn investigated with $\mathrm{C}$ - reactive protein and blood culture sensitivity. All babies (excluding those in criteria mentioned above) with score below 7 were started on BCPAP within 1 hour of birth (inborn) /admission (outborn) They were monitored for a period of 72 hours on Bubble CPAP.

After 72 hours, BCPAP was considered to be a success if the distress improved and baby could be wea4ned from CPAP with minimal retractions, Saturation of $>90 \%$ with $\mathrm{FiO}_{2}$ of $30 \%$, and PEEP of $5 \mathrm{~cm}$. Babies who had worsening of symptoms, remained hypoxic, with saturation of $<85 \%$, severe chest retractions, $\mathrm{FiO}_{2}$ requirement of more than $70 \%$, PEEP of $>7 \mathrm{~cm}$ with circulatory failure requiring inotropes etc., were considered for failure of CPAP and were intubated and connected to Mechanical Ventilator. These criteria were drawn based on the protocols followed in premier institutes across the country. Outcome was determined at the end of 72 hours based on studies done in the past with BCPAP ${ }^{[11]}$

The indigenous Bubble CPAP system that was used, had a source of gas flow, binasal prongs adjusted according to head circumference of baby, a respiratory circuit for the gas flow, a wide bore expiratory tube, whose end was inserted in a bottle of water or normal saline. The PEEP provided by BCPAP is indicated by the distance to which the expiratory tube was submerged underwater., For eg., if the tube was inserted $5 \mathrm{~cm}$ below the water, $5 \mathrm{~cm}$ of PEEP was being provided to the neonate. Oxygen flow was adjusted between 4-6 1/min.

As the primary outcome, the study compared, the proportion of success or failure, on early initiation of bubble CPAP. The other outcomes that were studied are predictors associated with good clinical outcome, influence on factors such as age, gestational age, causative factors for respiratory distress on Downes score and its improvement following BCPAP, correlation of Downes' score with clinical outcome and duration of stay in hospital and Association of BCPAP initiation with clinical outcome (Table 2)

Data were analysed using Fischer exact test for comparison of proportions and independent ' $t$ ' test to compare means between two groups. $P$ value $<0.05$ was considered as significant.

Table 1: Type of neonates in study

\begin{tabular}{|c|c|}
\hline Type & $\mathbf{N}(\mathbf{\%})$ \\
\hline Males & $91(60.7 \%)$ \\
\hline Females & $59(39.3 \%)$ \\
\hline Term babies (>37 weeks) & $87(58 \%)$ \\
\hline Late preterm babies (34-36 weeks) & $35(23.3 \%)$ \\
\hline Early preterm babies (28-33 weeks) & $24(16 \%)$ \\
\hline Extreme preterm (<28 weeks) & $4(2.7 \%)$ \\
\hline
\end{tabular}

\section{Results}

\section{Early Initiation}

The early initiation of Bubble CPAP within 1 hour in neonatal intensive care unit showed that out of 150 babies, $114(80.9 \%)$ babies survived, while $1(16.7 \%)$ baby expired and 2 babies $(66.7 \%)$ were referred. The mean Downes' Score on day 1in early initiation of Bubble CPAP was $2.58 \pm 1.62$ in comparison to those with late initiation which was $4.21 \pm 2.6$. This difference was statistically significant using $t$ test at $\mathrm{p}<0.001$ level. The mean Downes' Score on day 3in early initiation of Bubble CPAP was $0.76 \pm 1.27$ in comparison to those with late initiation which was2.07 \pm 2.3 . This difference was statistically significant using t test at $\mathrm{p}<0.001$ level. (Figure 1)

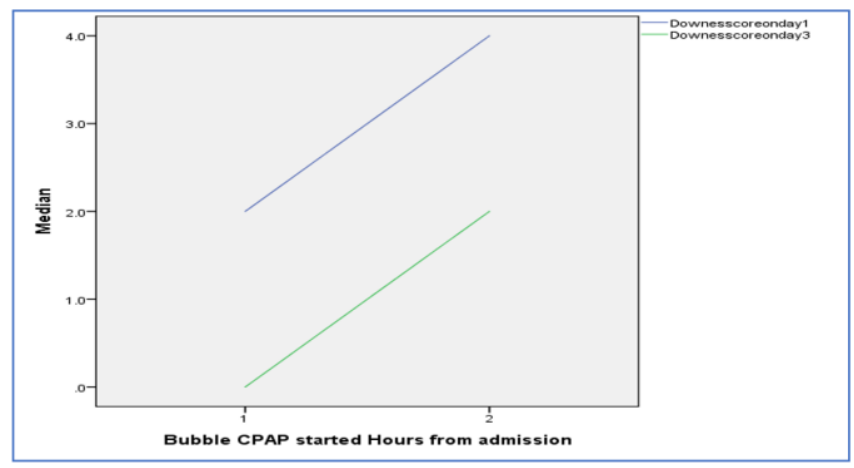

Fig 1: Association between Hours of initiation of Bubble CPAP with the Modified Downes' Score on Day $1 \&$ Day 3 of admission $(n=150)$

\section{Clinical outcome}

As a clinical outcome, survival was better among term babies. Amongst the enrolled 150 babies, 58\% of term babies, $15.3 \%$ of early preterm babies, $20 \%$ of late preterm 
babies and $0.7 \%$ of extremely preterm babies, admitted with respiratory distress survived. Death was not reported among term babies. The result was statistically significant at $\mathrm{p}<0.001$ level.

While comparing clinical outcome and birth weight of babies in BCPAP, $66 \%$ of babies beyond $2.5 \mathrm{~kg}$ survived better, while $28 \%$ of babies in the range of $0.6 \mathrm{~kg}-2.4 \mathrm{~kg}$ survived. Death occurred among $3.3 \%$ of low birth weight babies, $0.7 \%$ of normal weight babies. The result was statistically significant at $\mathrm{p}<0.001$.

Good Clinical Outcome was seen in $17.3 \%$ of Transient Tachypnoea of newborn (TTN), $2.7 \%$ of clinically proven sepsis, $27.3 \%$ of Meconium stained liquor (MSL) babies, $3.3 \%$ of bronchiolitis/pneumonia, $6.7 \%$ of Hypoxic ischemic encephalopathy (HIE) babies, $0.7 \%$ of apnea of prematurity and $36 \%$ of babies who had 2 or more attributable causes to respiratory distress eg., HIE with pneumonia etc., ion. The result was statistically significant at $\mathrm{p}<0.001$ level. There was no gender wise disparity noted among neonates with BCPAP.

\section{BCPAP and Downes score}

A total of $1.1 \%$ of term babies, $12.5 \%$ of early preterm babies, $2.9 \%$ of late preterm babies and $50.0 \%$ of extremely preterm babies had severe Downes score on Day 1 of admission. Out of 150 patients, $10 \%$ of low birth weight babies and $2 \%$ of babies with birth weight more than $2.5 \mathrm{~kg}$ found to have severe Downes' Score on Day 1 of admission. The result was statistically significant at $\mathrm{p}<0.01$ level. A total of $3.4 \%$ of term babies, $25 \%$ of early preterm babies, $14.3 \%$ of late preterm babies and $75.0 \%$ of extremely preterm babies had moderate Downes' Score on Day 3 of admission. The result was statistically significant at $\mathrm{p}<0.001$ level.

While out of the 150 patients, $32 \%$ of low birth weight babies and $4 \%$ of babies with birth weight more than $2.5 \mathrm{~kg}$ found to have moderate Modified Downes' Score on Day 3 of admission. The result was statistically significant at $\mathrm{p}$ $<0.001$ level.

Moderate category of Modified Downes score on Day 1 of admission was seen in $34.6 \%$ of TTN, $25.0 \%$ of sepsis, $9.8 \%$ of MSL, $20.0 \%$ of bronchiolitis/pneumonia, $20.0 \%$ of HIE, $40.0 \%$ of apnea of prematurity and $23.7 \%$ of others $*(2$ or more direct causes to respiratory distress) Severe category of Modified Downes' Score on Day 1 of admission was seen in $60.0 \%$ of apnea of prematurity and $6.8 \%$ of others * $(2$ or more direct causes to respiratory distress $)$ miscellaneous causes. Moderate category of Downes score on Day 3 of admission was seen in $3.8 \%$ of TTN, $2.4 \%$ of MSL, $10.0 \%$ of HIE, $100.0 \%$ of apnea of prematurity and $15.3 \%$ of miscellaneous causes. Severe category of Modified Downe's Score on Day 3 of admission was seen in $1.7 \%$ of others. $*(2$ or more direct causes to respiratory distress). The lower the Downes' score better was the clinical outcome which was statistically significant at $\mathrm{p}<0.001$ level. The higher the Downes' score longer was the stay in the hospital. (Figure $2 \& 3$ )

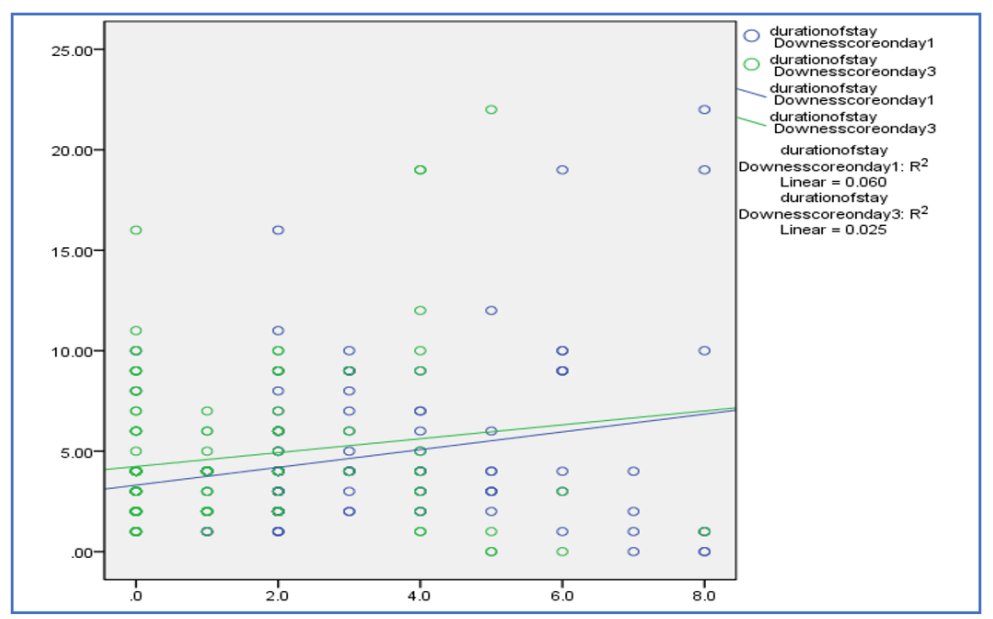

Fig 2: Correlation between Duration of stay with the Modified Downe's Score on Day $1 \&$ Day3 of admission ( $\mathrm{n}=150)$

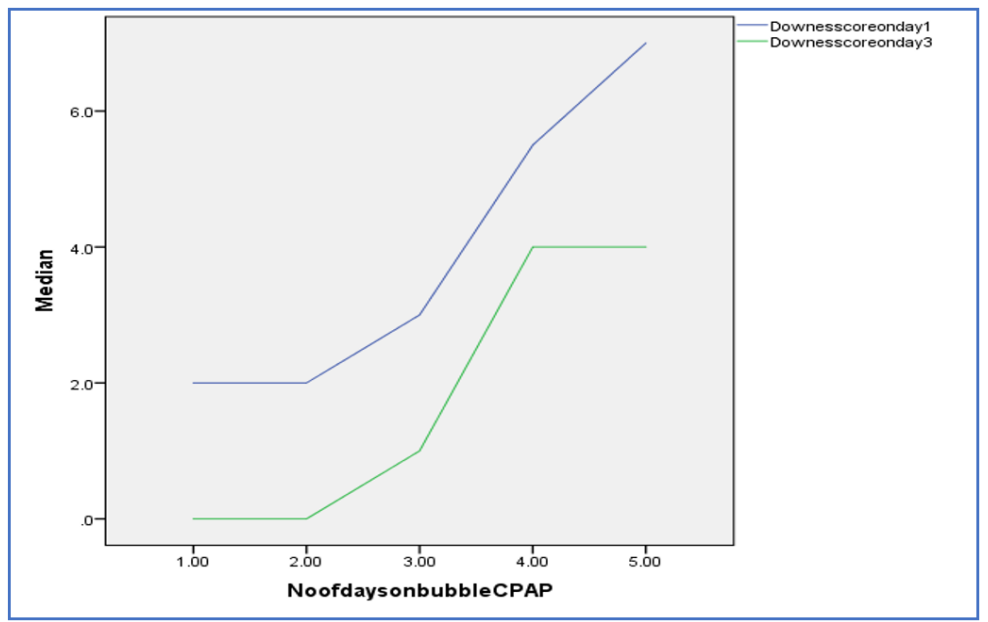

Fig 3: Association between Number of days on Bubble CPAP with the Modified Downes' Score on Day 1 \& Day 3 of admission ( $n=150$ ) 
Table 2: Variables associated with successful outcome on Bubble CPAP

\begin{tabular}{|c|c|c|}
\hline Variable & $\begin{array}{l}\text { BCPAP success } \\
\text { with n value }(\%)\end{array}$ & $\begin{array}{c}\mathbf{p} \\
\text { value }\end{array}$ \\
\hline Birth weight $600 \mathrm{gm}$ to $2499 \mathrm{gm}$ & $42(28 \%)$ & \multirow{2}{*}{0.001} \\
\hline Birth weight $>2500 \mathrm{gm}$ & $99(66 \%)$ & \\
\hline Gestational age $>37$ weeks & $87(100 \%)$ & \multirow{4}{*}{0.001} \\
\hline Gestational age 34-36 weeks & $30(85.7)$ & \\
\hline Gestational age $28-33$ weeks & $23(95.8 \%)$ & \\
\hline Gestational age $<28$ weeks & $1(25 \%)$ & \\
\hline Initiation of BCPAP $<1$ hour & $114(80.9 \%)$ & \multirow{2}{*}{0.001} \\
\hline Initiation of BCPAP > 1 hour & $27(19.1 \%)$ & \\
\hline $\begin{array}{c}\text { Outcome with mild/moderate } \\
\text { category Downes score on Day } 1\end{array}$ & $\begin{array}{c}109(77.3 \%) \text {-mild } \\
28(19.9 \%) \text {-moderate }\end{array}$ & \multirow{2}{*}{0.001} \\
\hline $\begin{array}{c}\text { Outcome with mild/moderate } \\
\text { category Downes score on Day } 3\end{array}$ & $\begin{array}{c}131(92.9 \%) \text {-mild } \\
10(7.1 \%) \text {-moderate }\end{array}$ & \\
\hline
\end{tabular}

\section{Discussion}

Similar to other studies and articles in neonatal ventilation, our study showed that early initiation of BCPAP improves respiratory distress and overall survival of newborn. Statistically significant success was observed among term, late and early preterm neonates, thus proving that BCPAP was effective beyond 28 weeks. In infants who weighed more than $1000 \mathrm{~g}$, BCPAP was found to be effective. BCPAP improved the Downes score and overall stay in hospital, thus reducing economic burden.

Since Mechanical ventilators require huge investment interms of equipment and training and with increasing evidence of complications due to non availability of skilled trainers in neonatal resuscitation, BCPAP could be effectively used especially in low income countries even at the level of primary and secondary care institutions, Thus BCPAP can be tried as a primary mode of support in resource poor settings ${ }^{[13]}$. significant reduction of mortality following introduction of BCPAP suggested that implementation of this practice at primary health care level would yield good result as this technique had fewer adverse outcomes ${ }^{[14]}$.

\section{Limitations}

This is a single centre study. The neonates included in our study belonged to different gestational maturity groups and early preterms who required surfactant were not included in our study as INSURE technique is a popular reason for using BCPAP these days. 3 neonates developed pneumothorax, while few babies were referred in view of parental concerns, expert management etc., Few studies point that neonates may be subjected to excessive exposure to oxygen and nosocomial infection while using $\mathrm{FiO}_{2}>70 \%$ and used nasal prongs, which has to be explored further ${ }^{[15}$, ${ }^{17]}$. Usage of Oxygen and implication on bronchopulmonary dysplasia, retinopathy of prematurity and neurodevelopmental outcome should also be reviewed ${ }^{[18]}$. Case fatality rates for neonates receiving BCPAP are higher in India than in other countries, and complications might be under-reported $^{[15]}$.

\section{References}

1. The WHO Reproductive Health Library https://extranet.who.int/rhl/topics/newborn-health/airversus-oxygen-resuscitation-infants-birth,accessed on 1.11.20

2. World Health Organization. Who recommendations on interventions to improve preterm birth outcomes.
Geneva: World Health Organization, 2015.

3. Gregory GA, Kitterman JA, Phibbs RH et al. Treatment of the idiopathic respiratory-distress syndrome with continuous positive airway pressure. N Engl J Med 1971;284:1333-40.

4. Avery ME, Tooley WH, Keller JB et al. Is chronic lung disease in low birth weight infants preventable? A survey of eight centers. Pediatrics 1987;79:26-30.

5. Sonja Baldursdottir, Markus Falk, Snorri Donaldsson, Baldvin Jonsson, Thomas Drevhammar. Basic principles of neonatal bubble CPAP: effects on CPAP delivery and imposed work of breathing when altering the original design.

6. Thomson MA, Yoder BA, Winter VT, Giavedoni L, Chang LY, Coalson JJ. Delayed extubation to nasal continuous positive airway pressure in the immature baboon model of Broncho pulmonary dysplasia: lung clinical and pathological findings. Pediatrics 2006;118:2038-50. doi:10.1542/peds.2006-0622

7. Pillow JJ, Hillman N, Moss TJM, Polglase G, Bold G, Beaumont $\mathrm{C}$ et al. Bubble continuous positive airway pressure enhances lung volume and gas exchange in preterm lambs. Am J Respir Crit Care Med 2007;176:63-9. doi:10.1164/rccm.2006 09- b b13680C

8. Lee KS, Dunn MS, Fenwick M, Shennan AT. A comparison of underwater bubble continuous positive airway pressure with ventilator derived continuous positive airway pressure in premature neonates ready for extubation. Biol Neonate 1998;73:69-75. doi: 10.1159

9. Rossano Rezzonico, Letizia Caccamo M, Valeria Manfredini, Massimo Cartabia, Nieves Sanchez, Zoraida Paredes et al. Impact of the systematic introduction of low-cost bubble nasal CPAP in a NICU of a developing country: a prospective pre- and postintervention study, 2015.

10. Walufu Ivan Egesa, William Mugowa Waibi. Bubble Nasal Continuous Positive Airway Pressure (bNCPAP): An Effective Low-Cost Intervention for ResourceConstrained Settings, 2020.

11. Ammari A, Suri M, Milisavljevic V, Sahni R, Bateman $\mathrm{D}$, Sanocka U et al. Variables associated with the early failure of nasal CPAP in very low birth weight infants. J Pediatr. 2005;147:341-347.

12. Jagdish Koti, Srinivasmurki, Pramodgaddam, Anupamareddy Andm Dasaradha Rami Reddy. bubble cpap for respiratory distress syndrome in preterm infants, 2009.

13. Thukral A, Sankar MJ, Chandrasekaran A, Agarwal R, Paul VK. Efficacy and safety of CPAP in low- and middle-income countries, 2016.

14. Juan Emmanuel Dewez, Sushma Nangia, Harish Chellani, Sarah White, Matthews Mathai, Nynke van den. Broek-Healthcare workers' views on the use of continuous positive airway pressure (CPAP) in neonates: a qualitative study in Andhra Pradesh, India, 2019.

15. Juan Emmanuel Dewez, Sushma Nangia, Harish Chellani, Sarah White, Matthews Mathai, Nynke van den. Broek-Healthcare workers' views on the use of continuous positive airway pressure (CPAP) in neonates: a qualitative study in Andhra Pradesh, India, 2019.

16. Ibrahim Sammour, Sreenivas Karnati. Non-invasive 
Respiratory Support of the Premature Neonate: From Physics to Bench to Practice, 2020.

17. Shruti Bharadwaj K, Abdullah Alonazi, Laura Banfield, Sourabh Dutta. Bubble versus other continuous positive airway pressure forms, 2020

18. Van Marter LJ, Allred EN, Pagano M et al. Do clinical markers of barotrauma and oxygen toxicity explain interhospital variation in rates of chronic lung disease? the neonatology Committee for the developmental network, Pediatrics. 